\title{
Consumo de álcool por acadêmicos de medicina de uma Universidade Pública no
}

\section{Estado do Pará}

\author{
Alcohol consumption by medical students at a Public University in the State of Pará \\ Consumo de alcohol por estudiantes de medicina de una Universidad Pública del Estado de Pará
}

Recebido: 28/02/2021 | Revisado: 18/03/2021 | Aceito: 20/03/2021 | Publicado: 27/03/2021

Iris Silva Rodrigues

ORCID: https://orcid.org/0000-0002-6956-8847 Universidade Federal do Pará, Brasil

E-mail: íris_silva_rodrigues@hotmail.com

Ana Beatriz Passos Nunes Carvalho

ORCID: https://orcid.org/0000-0002-6546-6130 Universidade Federal do Pará, Brasil E-mail: biamedufpa@gmail.com

Jéssyca Amanda Gomes Medeiros ORCID: https://orcid.org/0000-0003-1838-5298 Universidade Federal do Pará, Brasil E-mail: jessycamandag@gmail.com João Victor Moura Garcia

ORCID: https://orcid.org/0000-0001-6305-6042 Universidade Federal do Pará, Brasil E-mail: joaovgarcia98@gmail.com

Izaura Maria Vieira Cayres Vallinoto

ORCID: https://orcid.org/0000-0003-1408-8384 Universidade Federal do Pará, Brasil E-mail: ivallinoto@ufpa.br

\begin{abstract}
Resumo
Objetivo: avaliar a prevalência do consumo de álcool pelos estudantes regularmente matriculados na faculdade de medicina da Universidade Federal do Pará (UFPA), a fim de traçar um perfil socioeconômico e do padrão de consumo de álcool dos acadêmicos de medicina de acordo com o semestre em andamento Métodos: estudo retrospectivo, descritivo, transversal com abordagem quantitativa para avaliar diversos aspectos do perfil de consumo de álcool e socioeconômico de 760 estudantes na Faculdade de Medicina da UFPA. Resultados: nosso estudo revelou uma prevalência de $71,2 \%$ do consumo de álcool, de $51,2 \%$ do sexo masculino; da faixa etária entre 18 a 25 anos de idade $(79,8 \%)$; do estado civil solteiro $(95,6 \%)$; do catolicismo como religião $(57,7 \%)$; da naturalidade da região metropolitana de Belém (55,8\%); da moradia com familiares (73,3\%); de renda familiar renda menor que cinco mil reais $(47,7 \%)$; da maior ingestão de bebidas alcoólicas por estudantes dos primeiros dois anos do curso e da zona de risco I de acordo com a pontuação obtida no Teste de Identificação de Transtornos Devido ao Álcool (AUDIT). 91\% dos estudantes afirmaram consumir álcool em uma frequência de até quatro vezes ao mês. $\mathrm{O}$ perfil dos nossos entrevistados em relação a sinais e sintomas de dependência ao álcool não apresentou graves problemas. Conclusão: a prevalência do consumo de álcool encontrada neste estudo é preocupante e exige atenção da comunidade acadêmica. Palavras-chave: Consumo de álcool na faculdade; Estudantes de medicina; Prevalência; Alcoolismo; Transtornos Relacionados ao uso de álcool.
\end{abstract}

\begin{abstract}
Objective: available the prevalence of alcohol consumption by students regularly enrolled in the medical school of the Federal University of Pará (UFPA), to outline a socioeconomic profile and the pattern of alcohol consumption among medical students according to the current semester. Methods: a retrospective, descriptive, cross-sectional study with a quantitative approach to assess various aspects of the alcohol and socioeconomic profile of 760 students at the Faculty of Medicine at UFPA. Results: our study revealed a prevalence $71.2 \%$ of alcohol consumption, $51.2 \%$ of males; the age group between 18 to 25 years old (79.8\%); single marital status (95.6\%); Catholicism religion (57.7\%); the naturalness of metropolitan region of Belém (55.8\%); housing with family members (73.3\%); family income less than five thousand reais (47.7\%); the highest intake of alcoholic beverages by students in the two first years the course and the risk zone I, according to the score obtained in Alcohol Use Disorder Identification Test (AUDIT). 91\% of students said they consume alcohol at a frequency of up to four times a month. Our interviewees did not present any serious problem when we regarded the signs and symptoms of alcohol dependence. Conclusion: the prevalence of
\end{abstract}


alcohol consumption found in this study is worrying and requires attention from the academic community. Keywords: Alcohol drinking in college; Students, medical; Prevalence; Alcoholism; Alcohol-related disorders.

\begin{abstract}
Resumen
Objetivo: evaluar la prevalencia de consumo de alcohol por estudiantes matriculados regularmente en la facultad de medicina de la Universidad Federal de Pará (UFPA), con el fin de delinear un perfil socioeconómico y el patrón de consumo de alcohol de los estudiantes de medicina según el semestre en curso. Métodos: estudio retrospectivo, descriptivo, transversal con abordaje cuantitativo para evaluar diversos aspectos del perfil alcohólico y socioeconómico de 760 estudiantes de la Facultad de Medicina de la UFPA. Resultados: nuestro estudio reveló una prevalencia del $71,2 \%$ de consumo de alcohol, del 51,2\% de los hombres; el grupo de edad entre 18 y 25 años (79,8\%); estado civil soltero (95,6\%); El catolicismo como religión (57,7\%); la naturalidad de la región metropolitana de Belém (55,8\%); vivienda con familiares (73,3\%); ingresos familiares inferiores a cinco mil reales $(47,7 \%)$; la mayor ingesta de bebidas alcohólicas por parte de los estudiantes en los dos primeros años del curso y la zona de riesgo I según la puntuación obtenida en Prueba de Identificación de Trastornos por Alcohol (AUDIT). El 91\% de los estudiantes dijeron que consumen alcohol con una frecuencia de hasta cuatro veces al mes. El perfil de nuestros entrevistados en cuanto a signos y síntomas de dependencia del alcohol no presentó problemas graves. Conclusión: la prevalencia de consumo de alcohol encontrada en este estudio es preocupante y requiere atención por parte de la comunidad académica.
\end{abstract}

Palabras clave: Consumo de alcohol em la universidade; Estudiantes de medicina; Prevalencia; Alcoholismo; Transtornos relacionados com alcohol.

\title{
1. Introdução
}

O álcool é a substância psicoativa mais consumida do mundo (Bray et al., 2018). Mais de 2,3 bilhões de pessoas no mundo consomem bebidas alcoólicas e 283 milhões apresentam algum nível de dependência (WHO, 2018). Além disso, a Organização Mundial da Saúde (OMS) (2018) aponta o álcool como responsável por uma das maiores taxas de mortalidade, três milhões de mortes por ano, 5,3\% do total de mortes, maior que as causadas por doenças como tuberculose, HIV/AIDS e diabetes.

No Brasil, o consumo de álcool no país é bem estabelecido, cerca de 17,9\% da população faz uso abusivo de bebidos alcoólicas, as quais são responsáveis por cerca de 1,45\% de óbitos (Brasil, 2019). Segundo o II Levantamento Nacional de Álcool e Drogas (LENAD), 52\% dos brasileiros adultos bebem (UNIAD, 2012). A prevalência da ingestão de álcool é maior em homens (65\%), no entanto há um crescimento de 42,9\% entre as mulheres no país (Brasil, 2019).

O consumo de bebidas alcoólicas é amplamente aceito e incentivado na sociedade atual (Bray et al., 2018; Galduróz, Noto, Nappo \& Carlini, 2005). A maioria da juventude inicia o consumo de álcool antes dos 15 anos de idade e a maior prevalência dos consumidores estão entre jovens 15 e 24 anos (Bray et al., 2018). O Centro Brasileiro de Informações sobre Drogas Psicotrópicas (CEBRID) afirma que o álcool tem sido a substância mais procurada por adolescentes e cerca de $86,4 \%$ dos estudantes acima de 19 anos de idade fazem uso de bebida alcoólica em algum grau (Carlini, 2011; UNIAD, 2012). Sabese que quanto mais precoce é o início do uso do álcool, maior a propensão para se estabelecer uma dependência (Pelicioli, Barelli, Gonçalves, Hahn \& Scherer, 2017). Desse modo, a população jovem apresenta maior vulnerabilidade a apresentar dependência e consequências negativas à saúde (Pelicioli, Barelli, Gonçalves, Hahn \& Scherer, 2017; Carvalho, Heilig, Perez, Probst \& Rehm, 2019).

O consumo moderado de álcool é um fator de proteção para doenças cardiovasculares (Le Daré, Lagente \& Gicquel, 2019). Todavia, é uma substância psicoativa largamente utilizada por produzir sensações prazerosas no indivíduo e, consequentemente, consumida em excesso no meio social (Becker, 2017)' (Galduróz, Noto, Nappo \& Carlini, 2005). Binge Drinking (beber em excesso) é o termo utilizado para caracterizar eventos de consumo de cinco ou mais doses de álcool em uma mesma ocasião, sendo 1 dose equivalente a, aproximadamente, $350 \mathrm{~mL}$ de cerveja, a $150 \mathrm{~mL}$ de vinho ou a $40 \mathrm{~mL}$ de uma bebida destilada (Mourão, Andrade, Marques, Aparecida \& Prates, 2015). Vale destacar que Binge Drinking difere do 
alcoolismo, uma vez que este é um transtorno marcado pelo uso crônico e excessivo de álcool, resultando em problemas psicológicos, interpessoais e médicos (Spanagel, 2018).

A ingestão excessiva de bebidas alcoólicas pode causar mais de 200 tipos de distúrbios fisiológicos, principalmente transtornos psicossociais, haja vista que o álcool é uma droga psicotrópica e tem alta atração pelo sistema nervoso central (SNC) (Carlini, 2011; Carvalho, Heilig, Perez, Probst \& Rehm, 2019; Le Daré, Lagente \& Gicquel, 2019). Além disso, o Binge Drinking também tem sido associado a diversos problemas sociais, como baixo desempenho acadêmico, violência e uso de múltiplas drogas (Gomes et al., 2018; Zeferino et al., 2015).

Em vista disso, a OMS criou o Teste de Identificação de Transtornos Devido ao Álcool (AUDIT), um questionário de dez questões para avaliar o consumo de risco ou nocivo de álcool (Babor, Higgins-Biddle, Saunders \& Monteiro, 2001; Santos, Gouveia, Fernandes, Souza \& Grangeiro, 2012). Esse teste revelou-se instrumento confiável para o levantamento de informações e apresentou excelente consistência interna, sendo utilizado no mundo inteiro (Mattara, Ângelo, Faria \& Campos, 2010). No Brasil, o AUDIT tem sido amplamente utilizado em diversos campos, principalmente aqueles referentes ao âmbito universitário, no qual possibilita a abordagem da população mais susceptível aos danos da ingestão de bebidas alcoólicas (Santos, Gouveia, Fernandes, Souza \& Grangeiro, 2012; Mattara, Ângelo, Faria \& Campos, 2010).

O curso de medicina é a área acadêmica na qual os estudantes estão mais vulneráveis ao uso abusivo de bebidas alcoólicas (Mourão, Andrade, Marques, Aparecida \& Prates, 2015). O processo de ingresso na universidade, associado à mudança para a vida adulta, na qual o jovem necessita assumir responsabilidades e ao excesso de cargas horárias na grade curricular pode desencadear altos níveis de estresse, de ansiedade e de sintomas depressivos, levando a busca do consumo de álcool como mecanismo de fuga (Gomes et al., 2018; Becker, 2017).

Diante disso, este estudo tem como objetivo avaliar a prevalência do consumo de álcool pelos estudantes regularmente matriculados na faculdade de medicina da Universidade Federal do Pará, a fim de traçar um perfil socioeconômico e do padrão de consumo de álcool dos acadêmicos de medicina de acordo com o semestre em andamento.

\section{Metodologia}

Trata-se de um estudo retrospectivo, descritivo, transversal com abordagem quantitativa, que, segundo Pereira, Shitsuka, Parreira e Shitsuka (2018), é realizada a coleta de dados quantitativos ou numéricos por meio de medições de grandezas e obtém-se por meio da metrologia, números com suas respectivas unidades, e que posteriormente podem ser analisadas através de técnicas matemáticas como porcentagem, estatísticas e probabilidades além de poder ser empregados métodos analíticos e métodos numéricos. O estudo foi desenvolvido na Faculdade de Medicina da Universidade Federal do Pará, para avaliar diversos aspectos do perfil de consumo de álcool e socioeconômico de 760 estudantes matriculados, provenientes de todos os períodos do curso.

Foram convidados a participar do estudo todos os alunos disponíveis e, por conveniência, sem amostragem probabilística ou aleatorizada. Foram incluídos na pesquisa indivíduos de ambos os sexos, regularmente matriculados do primeiro ao décimo segundo períodos e que concordaram em participar da pesquisa. Foram excluídos da pesquisa os alunos menores de 18 anos, que não preencheram o Termo de Consentimento Livre e Esclarecido, cujos questionários foram preenchidos de maneira inadequada ou que não concordaram em participar do estudo.

Todos os indivíduos da presente pesquisa foram estudados segundo os preceitos da Declaração de Helsinque e do Código de Nuremberg, respeitadas as Normas de Pesquisa Envolvendo Seres Humanos (Resolução CNS 196/96) do Conselho Nacional de Saúde após a submissão e aprovação do projeto pelo Comitê de Ética em Pesquisa com Seres Humanos do Instituto de Ciências da Saúde da Universidade Federal do Pará em 31 de janeiro de 2020, sob parecer número 3.817.351. 
A coleta de dados foi realizada durante os meses de fevereiro e de março do ano de 2020. Os dados foram coletados pelos autores através dos questionários AUDIT (Babor, Higgins-Biddle, Saunders \& Monteiro, 2001) e de perfil socioeconômico. Foram registradas as seguintes variáveis com relação aos estudantes: gênero, idade, naturalidade, semestre em andamento, religião, estado civil, tipo de moradia, renda familiar e atividade profissional. Quando o estudante não preenchia o espaço de religião foi considerado nenhuma. Na avaliação do perfil de consumo alcoólico pelo AUDIT foram avaliadas as seguintes variáveis: frequência do consumo, dependência e consequências negativas do consumo de álcool.

Os dados obtidos foram armazenados em planilhas eletrônicas usando o software Microsoft Office Excel 2010. Após serem computados, os dados foram analisados através do pacote estatístico Bioestat 5.0. A análise univariada da significância dos dados foi realizada pelo teste do Qui-quadrado de Aderência. Valores de $\mathrm{p}<0,0001$ indicam significância estatística.

\section{Resultados}

A população foi composta por um total de 760 acadêmicos do curso de Medicina, dos quais $71,2 \%$ declararam consumir bebidas alcoólicas (etilistas). Segundo as características socioeconômicas levantadas (Tabela 1), as variáveis faixa etária, estado civil, religião, moradia e renda familiar apresentaram diferença significativa ( $p$ valor $<0,0001$ ) entre os fatores avaliados. Em análise do perfil socioeconômico dos acadêmicos etilistas, observou-se maior prevalência do sexo masculino (51,2\%); da faixa etária de acadêmicos entre 18 a 25 anos de idade (79,8\%); do estado civil solteiro (95,6\%); do catolicismo como religião (57,7\%); naturalidade da região metropolitana de Belém (55,8\%); moradia com familiares (73,3\%); de renda familiar menor que cinco mil reais $(47,7 \%)$. Em relação ao período de curso, as três maiores prevalências foram, quarto $(11,3 \%)$, terceiro $(9,6 \%)$ e décimo segundo $(9,6 \%)$ períodos. 
Tabela 1 - Perfil socieconômico dos acadêmicos etilistas de medicina da Universidade Federal do Pará - UFPA, 2020.

\begin{tabular}{|c|c|c|}
\hline Variáveis & $\begin{array}{c}\text { Etilista } \\
\mathrm{N}=541 \\
\mathrm{n}(\%)\end{array}$ & p valor* \\
\hline \multicolumn{3}{|l|}{ Sexo } \\
\hline Masculino & $277(51,2)$ & \multirow{2}{*}{0,6059} \\
\hline Feminino & $264(48,8)$ & \\
\hline \multicolumn{3}{|l|}{ Faixa etária (anos) } \\
\hline $18-25$ & $432(79,8)$ & \multirow{4}{*}{$<0,0001$} \\
\hline $26-33$ & $94(17,4)$ & \\
\hline $34-41$ & $15(2,8)$ & \\
\hline $42-49$ & 0 & \\
\hline \multicolumn{3}{|l|}{ Período do curso } \\
\hline Quarto & $61(11,3)$ & \multirow{3}{*}{0,1499} \\
\hline Terceiro & $52(9,6)$ & \\
\hline Décimo segundo & $52(9,6)$ & \\
\hline \multicolumn{3}{|l|}{ Estado civil } \\
\hline Solteiro & $517(95,6)$ & \multirow{3}{*}{$<0,0001$} \\
\hline Casado & $17(3,1)$ & \\
\hline União estável & $7(1,3)$ & \\
\hline \multicolumn{3}{|l|}{ Religião } \\
\hline Católica & $312(57,7)$ & \multirow{4}{*}{$<0,0001$} \\
\hline Evangélica & $40(7,4)$ & \\
\hline Outra religião & $65(12)$ & \\
\hline Nenhuma & $124(22,9)$ & \\
\hline \multicolumn{3}{|c|}{ Naturalidade região metropolitana de Belém-PA } \\
\hline Sim & $302(55,8)$ & \multirow{2}{*}{0,0077} \\
\hline Não & $239(44,2)$ & \\
\hline \multicolumn{3}{|l|}{ Moradia } \\
\hline Familiar & $396(73,3)$ & \multirow{4}{*}{$<0,0001$} \\
\hline Amigo(a) & $69(12,6)$ & \\
\hline Sozinho(a) & $67(12,4)$ & \\
\hline Pensionato / república & $9(1,7)$ & \\
\hline \multicolumn{3}{|l|}{ Renda familiar } \\
\hline Menos de cinco mil reais & $258(47,7)$ & \multirow{4}{*}{$<0,0001$} \\
\hline Entre cinco a dez mil reais & $153(28,3)$ & \\
\hline Acima de dez mil reais & $75(13,9)$ & \\
\hline Não soube informar & $55(10,1)$ & \\
\hline
\end{tabular}

* Qui-quadrado. Fonte: Protocolo de Pesquisa (2020).

Em análise ao perfil dos acadêmicos etilistas relacionados a fatores de quantidade, de frequência e de sintomas de dependência de bebidas alcoólicas (Tabela 2), observou-se maior prevalência da ingestão de bebidas alcoólicas de duas a quatro vezes por mês $(45,7 \%)$ e de uma vez por mês ou menos $(45,3 \%)$, sendo que, em um dia normal, esse consumo é maior do que uma ou duas bebidas $(65,4 \%)$. Ao questionar, a frequência com que consomem seis bebidas ou mais numa única ocasião, 39,7\% dos alunos afirmam que consomem em menos de uma vez por mês. Ao questionar os últimos 12 meses com relação à frequência que se "percebeu de que não conseguia parar de beber depois de começar", 76,9\% dos acadêmicos etilistas afirmaram que tal fato nunca aconteceu. No mesmo período de 12 meses, a maior prevalência do número de acadêmicos etilistas que "não conseguiu cumprir as tarefas que habitualmente lhe exigem por ter bebido", foi de nunca terem passado por essa situação (76,2\%) e a condição de "precisou beber logo de manhã para "curar" uma ressaca", foi de nunca terem passaram por isso (a maior prevalência). 
Tabela 2 - Distribuição com relação à quantidade, à frequência e aos sintomas de dependência de bebidas alcoólicas pelos acadêmicos de medicina considerados etilistas da Universidade Federal do Pará - UFPA, 2020.

\begin{tabular}{|c|c|c|}
\hline Variáveis & $\begin{array}{c}\text { Etilista } \\
\mathrm{N}=541 \\
\mathrm{n}(\%)\end{array}$ & p valor* \\
\hline \multicolumn{3}{|c|}{ Com que frequência consome bebidas que contêm álcool? } \\
\hline Nunca & $4(0,7)$ & \multirow{5}{*}{$<0,0001$} \\
\hline Uma vez por mês ou menos & $245(45,3)$ & \\
\hline Duas a quatro vezes por mês & $247(45,7)$ & \\
\hline Duas a três vezes por semana & $40(7,4)$ & \\
\hline Quatro ou mais vezes por semana & $5(0,9)$ & \\
\hline \multicolumn{3}{|c|}{$\begin{array}{l}\text { Quando bebe, quantas bebidas contendo álcool consome num dia } \\
\text { normal? }\end{array}$} \\
\hline Uma ou duas & $187(34,6)$ & \multirow{5}{*}{$<0,0001$} \\
\hline Três ou quatro & $134(24,8)$ & \\
\hline Cinco ou seis & $131(24,2)$ & \\
\hline De sete a nove & $54(9,9)$ & \\
\hline Dez ou mais & $35(6,5)$ & \\
\hline \multicolumn{3}{|c|}{$\begin{array}{l}\text { Com que frequência consome seis bebidas ou mais numa única } \\
\text { ocasião? }\end{array}$} \\
\hline Nunca & $186(34,5)$ & \multirow{5}{*}{$<0,0001$} \\
\hline Menos de um vez por mês & $215(39,7)$ & \\
\hline Pelo menos uma vez por mês & $109(20,1)$ & \\
\hline Pelo menos uma vez por semana & $30(5,5)$ & \\
\hline Diariamente ou quase diariamente & $1(0,2)$ & \\
\hline \multicolumn{3}{|c|}{$\begin{array}{l}\text { Nos últimos } 12 \text { meses, com que frequência se apercebeu de que não } \\
\text { conseguia parar de beber depois de começar? }\end{array}$} \\
\hline Nunca & $416(76,9)$ & \multirow{5}{*}{$<0,0001$} \\
\hline Menos de uma vez por mês & $76(14), 0$ & \\
\hline Pelo menos uma vez por mês & $41(7,6)$ & \\
\hline Pelo menos uma vez por semana & $7(1,3)$ & \\
\hline Diariamente ou quase diariamente & $1(0,2)$ & \\
\hline \multicolumn{3}{|c|}{$\begin{array}{l}\text { Nos últimos } 12 \text { meses, com que frequência não conseguiu cumprir as } \\
\text { tarefas que habitualmente lhe exigem por ter bebido? }\end{array}$} \\
\hline Nunca & $412(76,2)$ & \multirow{5}{*}{$<0,0001$} \\
\hline Menos de uma vez por mês & $99(18,3)$ & \\
\hline Pelo menos uma vez por mês & $25(4,6)$ & \\
\hline Pelo menos uma vez por semana & $4(0,7)$ & \\
\hline Diariamente ou quase diariamente & $1(0,2)$ & \\
\hline \multicolumn{3}{|c|}{$\begin{array}{l}\text { Nos últimos } 12 \text { meses, com que frequência precisou beber logo de } \\
\text { manhã para "curar" uma ressaca? }\end{array}$} \\
\hline Nunca & $499(92,2)$ & \multirow{5}{*}{$<0,0001$} \\
\hline Menos de uma vez por mês & $28(5,2)$ & \\
\hline Pelo menos uma vez por mês & $10(1,9)$ & \\
\hline Pelo menos uma vez por semana & $4(0,7)$ & \\
\hline Diariamente ou quase diariamente & 0 & \\
\hline
\end{tabular}

* Qui-quadrado. Fonte: Protocolo de Pesquisa (2020).

Já em análise ao perfil dos acadêmicos etilistas com relação à existência de problemas recentes com o consumo de bebidas alcoólicas (Tabela 3), observou-se a prevalência de sentimento de culpa ou de remorso nos últimos doze meses $(63,2 \%)$. A questão de "não se lembrou do que aconteceu na noite anterior por causa de ter bebido", mais da metade (61,6\%) garantiram sempre lembrar. Além disso, 80,8\% acadêmicos negaram ter já causado prejuízos a eles ou a pessoas após terem bebido, assim como 78,6\% denegam manifestarem preocupação em outras pessoas por causa do consumo de álcool. 
Tabela 3 - Distribuição com relação à existência de problemas recentes relacionados ao consumo de bebidas alcoólicas pelos acadêmicos de medicina considerados etilistas da Universidade Federal do Pará - UFPA, 2020.

\begin{tabular}{|c|c|c|}
\hline Variáveis & $\begin{array}{c}\text { Etilista } \\
\mathrm{N}=541 \\
\mathrm{n}(\%)\end{array}$ & p valor* \\
\hline \multicolumn{3}{|c|}{$\begin{array}{l}\text { Nos últimos } 12 \text { meses, com que frequência teve sentimento de culpa ou de remorso por ter } \\
\text { bebido? }\end{array}$} \\
\hline Nunca & $342(63,2)$ & \multirow{5}{*}{$<0,0001$} \\
\hline Menos de uma vez por mês & $146(27,0)$ & \\
\hline Pelo menos uma vez por mês & $40(7,4)$ & \\
\hline Pelo menos uma vez por semana & $12(2,2)$ & \\
\hline Diariamente ou quase diariamente & $1(0,2)$ & \\
\hline \multicolumn{3}{|c|}{$\begin{array}{l}\text { Nos últimos } 12 \text { meses, com que frequência não se lembrou do que aconteceu na noite } \\
\text { anterior por causa de ter bebido? }\end{array}$} \\
\hline Nunca & $333(61,6)$ & \multirow{5}{*}{$<0,0001$} \\
\hline Menos de uma vez por mês & $163(30,1)$ & \\
\hline Pelo menos uma vez por mês & $44(8,1)$ & \\
\hline Pelo menos uma vez por semana & 0 & \\
\hline Diariamente ou quase diariamente & $1(0,2)$ & \\
\hline \multicolumn{3}{|c|}{ Já alguma vez ficou ferido ou ficou alguém ferido por você ter bebido? } \\
\hline Não & $437(80,8)$ & \multirow{3}{*}{$<0,0001$} \\
\hline Sim, mas não nos últimos 12 meses & $63(11,6)$ & \\
\hline Sim, aconteceu nos últimos 12 meses & $41(7,6)$ & \\
\hline \multicolumn{3}{|c|}{$\begin{array}{l}\text { Já alguma vez um familiar, amigo, médico ou profissional de saúde manifestou preocupação } \\
\text { pelo seu consumo de álcool ou sugeriu que deixasse de beber? }\end{array}$} \\
\hline Não & $425(78,6)$ & \multirow{3}{*}{$<0,0001$} \\
\hline Sim, mas não nos últimos 12 meses & $47(8,7)$ & \\
\hline Sim, aconteceu nos últimos 12 meses & $69(12,7)$ & \\
\hline
\end{tabular}

* Qui-quadrado. Fonte: Protocolo de Pesquisa (2020).

Ao classificar o acadêmico etilista em uma de quatro zonas de risco de acordo com a pontuação obtida no AUDIT, percebeu-se que, independente do semestre do curso $\left(1^{\circ}-4^{\circ}, 5^{\circ}-8^{\circ}\right.$ e $9^{\circ}-12^{\circ}$ semestres), a maior frequência está concentrada na zona 1 (baixo risco) que indica uso de baixo risco ou abstinência e a menor frequência na zona 4 que sugere provável dependência (Gráfico 1). A frequência daqueles classificados como uso nocivo (zona 3) foi maior ao comparar com a zona 4, somente para aqueles que estão no 5-8 e 9-12 semestres. 
Gráfico 1 - Zonas de risco de acordo com o período do curso $\left(1^{\circ}\right.$ ao $4^{\circ}, 5^{\circ}$ ao $8^{\circ}$ e $9^{\circ}$ ao $12^{\circ}$ semestres $)$ de estudantes de medicina bebedores da Universidade Federal do Pará, Brasil, 2020.

Zona $1 \square$ Zona $2 \square$ Zona $3 \quad \square$ Zona 4

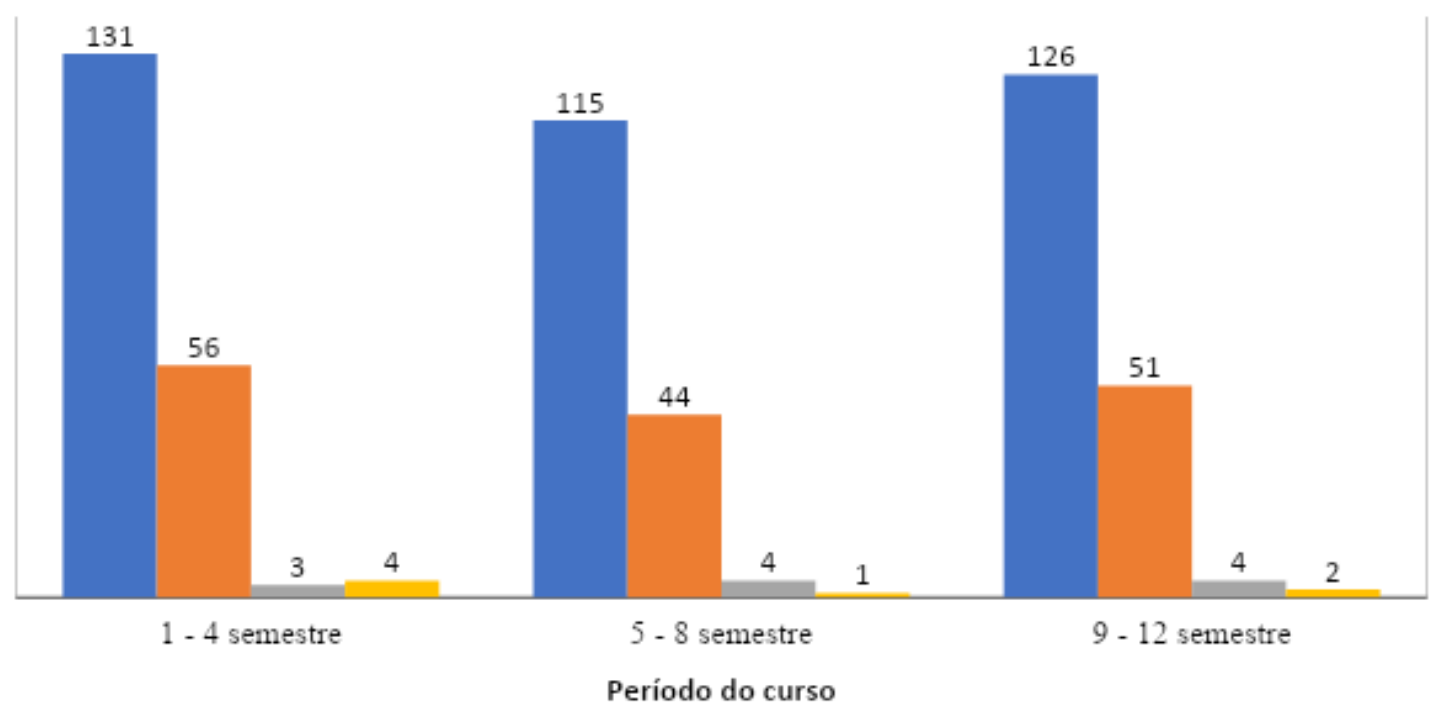

Fonte: Protocolo de Pesquisa (2020).

\section{Discussão}

Nosso estudo revelou uma alta prevalência do consumo de álcool entre os estudantes do curso de medicina da UFPA, tal como o perfil de consumo de álcool pela população brasileira $(86,2 \%)$ (Andrade, Duarte \& Oliveira, 2010; Pedrosa, Camacho, Passos \& Oliveira, 2011) e outros estudos brasileiros com universitários da área da saúde como os de Diadema-SP (77,6\%), de Passo Fundo-RS (85\%) e de Aracaju-SE (79,7\%) (Pelicioli, Barelli, Gonçalves, Hahn \& Scherer, 2017; Silva \& Tucci, 2014; Mendonça, Jesus \& Lima, 2018). Especificando para a realidade do curso de medicina, a literatura revela, também, a alta prevalência de modo mais acentuado, como entre estudantes de Medicina de Belo Horizonte (85,30\%) e de Jundiaí (91,20\%) (Ribeiro, França, Faria, Cuellar \& Martins, 2015; Amorim, Kikko, Abrantes \& Andrade, 2008; Spinelli, Valente \& Lotério, 2009). Diante disso, notam-se os acadêmicos de medicina como uma população vulnerável ao uso indevido de bebidas alcóolicas.

O perfil traçado no presente estudo apresenta maior prevalência de ingestão de bebidas alcoólicas por estudantes dos primeiros dois anos do curso de medicina, o qual é o período com excesso de conteúdo, densa carga horária de atividades curriculares, poucas horas de lazer e a fase de adaptação do aluno ao ambiente universitário (Becker, 2017; Gomes et al., 2018). Segundo Sompré (2019), os estudantes dos dois primeiros anos de graduação em medicina têm maior prevalência de depressão quando comparados aos demais períodos do curso. Em contrapartida, outro estudo observou um maior uso da substância com o decorrer da graduação (Barbosa et al., 2013). Tal divergência pode estar relacionada à distribuição curricular das disciplinas de forma particular em cada faculdade de Medicina do país. Todavia, em ambos os estudos é clara a forte relação entre a exposição a alto níveis de estresse e a busca pelo consumo de álcool.

Em análise ao perfil socioeconômico, vale destacar nossos resultados referentes às variáveis gênero, faixa etária, moradia e religião, haja vista que são fatores que podem influenciar em um maior de risco para dependência de substâncias alcoólicas (Mourão, Andrade, Marques, Aparecida \& Prates, 2015; Martins-Oliveira et al., 2016; Pelicioli, Barelli, Gonçalves, Hahn \& Scherer, 2017). 
Os estudantes do sexo masculino apresentaram-se como os maiores consumidores de bebidas alcoólicas, porém não houve diferença significativa entre os gêneros, isto é, os valores são tão próximos que não nos permite atribuir o gênero como um fator associado ao hábito de ingerir bebidas alcoólicas. Tal resultado pode ser reflexo do aumento do uso de álcool entre as mulheres na sociedade atual (Brasil, 2019; Gomes et al., 2013).

Estudos universitários atuais também demonstram esse processo de mudança do comportamento social, no qual, cada vez mais, é menor a diferença do etilismo entre os sexos (Mourão, Andrade, Marques, Aparecida \& Prates, 2015; Barbosa et al., 2013; Ramos \& Cunha, 2018). Vale ressaltar, que o organismo feminino necessita de uma menor quantidade de álcool para apresentar alguma problemática associada a essa substância (Erol \& Karpyak, 2015). Em vista disso, é notório que esse perfil de gêneros está associado a mudanças de comportamento psico-sócio-cultural, o que pode refletir, futuramente, em aumentos nos índices de prejuízos associados ao álcool.

A maior prevalência entre os estudantes etilistas do nosso estudo foi na faixa etária de 18-25 anos, semelhante as apresentadas pela OMS e pelo CEBRID como idade de maior índice de consumo de álcool (Bray et al., 2018; UNIAD, 2012; Carlini, 2011). Pesquisas que também investigaram perfil socioeconômico de acadêmicos de medicina etilista, também corroboram com o nosso achado (Ribeiro, França, Faria, Cuellar \& Martins, 2015; Assis \& Leão, 2016).

O início precoce do uso de substâncias psicoativas apresenta severas consequências para o organismo humano, como alterações cognitivas-comportamentais e desenvolvimento de doenças crônicas e inflamatórias (Carvalho, Heilig, Perez, Probst \& Rehm, 2019; Spear, 2018). Além disso, sabe-se que quanto mais precoce é o acesso ao álcool, maior o risco de apresentar algum grau de dependência (Spear, 2018; Pelicioli, Barelli, Gonçalves, Hahn \& Scherer, 2017). Diante disso, é preocupante que o consumo precoce de álcool entre universitários afete as atividades de desenvolvimento de excelência do futuro profissional da saúde.

Outros fatores socioeconômicos importantes na associação ao risco de consumo de álcool entre acadêmicos é moradia e religião, uma vez que estes afetam, diretamente, no comportamento social e na saúde mental do estudante (Becker, 2017; Martins-Oliveira et al., 2016). Todavia, os estudos presentes na literatura ainda são muito controversos, principalmente em relação à determinação dos fatores de risco ao etilismo, quando trata-se de relação familiar e de religiosidade (Zeferino et al., 2015; Assis \& Leão, 2016; Ramos \& Cunha, 2018). Desse modo, nota-se a importância, de investigar, mais atentamente, setores sociais altamente relacionados com a saúde mental dos jovens universitários.

Com relação à periodicidade do consumo, a quase totalidade dos estudantes de medicina da UFPA afirmaram consumir álcool em uma frequência de até quatro vezes ao mês, assim como uma predominância de um consumo de baixo risco. Tais achados se assemelham a estudos os quais revelam um baixo risco de consumo de bebidas alcóolicas entre universitários (Silva \& Tucci, 2014; Pelicioli, Barelli, Gonçalves, Hahn \& Scherer, 2017). Apesar do predomínio do consumo de baixo risco, quando, observam-se os dados obtidos em um dia normal, a maioria dos acadêmicos etilistas consomem mais de uma ou duas bebidas, embora o Binge Drinking (beber em excesso) seja caracterizado a partir da ingestão de cinco ou mais doses de álcool em uma mesma ocasião (Mourão, Andrade, Marques, Aparecida \& Prates, 2015), reafirmando que os nossos achados são um alerta para a ingestão excessiva de bebidas alcoólicas no meio universitário.

Embora já sabe-se que o consumo inadequado de substância psicotrópicas podem afetar nas atividades do cotidiano, devido a possíveis alterações nas funções mentais (Spear, 2018), no presente estudo não foram apresentados graves problemas, em relação a sinais e sintomas de dependência ao álcool, visto que mais da metade dos alunos nunca deixou de cumprir tarefas por ter bebido na noite anterior, não teve qualquer prejuízo na memória, não causou prejuízos a si ou a pessoas após terem bebido e não manifestou preocupação em outras pessoas por causa do consumo de álcool. O uso problemático do álcool por estudantes é frequentemente associado como gerador de problemáticas como, falta de atenção, sonolência em salas de aula, 
ausências, atrasos, saídas mais cedo das aulas e omissão no cumprimento dos compromissos (Baldisserotto, Filho, Nedel \& Sakae, 2005).

O ambiente universitário é um meio de alta carga de estresse, devido grande carga de compromisso curriculares e extracurriculares, assim como é meio de eventos sociais que oferecem de forma livre a venda da substância, tais como as festas do tipo open bar (bar aberto) frequentemente realizadas nos eventos esportivos. Dessa forma, destaca-se a importância de investimentos em trabalhos científicos que analisem graus de dependência ao álcool a partir da associação ao comportamento psicossocial no meio universitário, a fim de contribuir para a criação e direcionamento de afirmativas que atuem na prevenção à dependência química entre estudantes.

\section{Considerações Finais}

A prevalência do consumo de álcool encontrada neste estudo é preocupante e exige atenção por parte da equipe de docentes da faculdade de medicina da Universidade Federal do Pará, mas, principalmente, dos discentes da instituição tendo em vista que o jovem universitário logo estará no mercado de trabalho, sendo um formador de opiniões e promotor de educação em saúde para a sociedade.

Os resultados obtidos nesta e em outras pesquisas semelhantes de diversas faculdades de medicina do país mostram que esse tema merece ser melhor abordado por outras metodologias, pois se faz urgente e necessário alertar sobre os riscos do consumo dessa droga, por mais que ela seja legal e socialmente aceita em nosso meio.

Ademais, fica clara a necessidade de aproximação dos estudantes aos seus familiares e ao corpo docente da Universidade, uma vez que estes podem intervir, de forma direta, na problemática do álcool no meio estudantil. A inclusão de disciplinas sobre o uso abusivo e a dependência alcoólica ou de demais drogas, também pode ser uma proposta interessante, assim como a presença de apoio psicológico acessível dentro da faculdade que ajude no enfrentamento das dificuldades acadêmicas e pessoais.

Ainda que o estudo tenha demonstrado um elevado consumo alcoólico entre os acadêmicos analisados, é importante ressaltar que a população deste estudo é homogênea, representando somente os acadêmicos do curso de medicina, e por isso pode apresentar um perfil especifico do campo de estudo da pesquisa. Dessa forma, é desejável que novos estudos sejam realizados, preferencialmente multicêntricos, visando avaliar acadêmicos de outras graduações, a fim de se obter dados relevantes quanto a essa temática entre os diversos cursos de graduação, e a partir deles serem traçadas estratégias de apoio e acompanhamento desses estudantes dentro do ambiente universitário.

\section{Agradecimentos}

Agradecemos os acadêmicos de medicina da Universidade Federal do Pará pela participação no estudo.

\section{Referências}

Amorim, A. V. C., Kikko, E. O., Abrantes, M. M. \& Andrade V. L. A. (2008). Álcool e alcoolismo: estudo de prevalência entre discentes do curso de Medicina da UNIFENAS em Belo Horizonte-Minas Gerais. Rev Med Minas Gerais, 18:16-23.

Andrade, A. G., Duarte, P. C. A. V. \& Oliveira, L. G. (2010). I levantamento nacional sobre o uso de álcool, tabaco e outras drogas entre universitários das 27 capitais brasileiras. Brasília: Secretaria Nacional de Políticas sobre Drogas, 1:284. https://cetadobserva.ufba.br/sites/cetadobserva.ufba.br/files/634.pdf.

Assis, S. L. M. \& Leão, T. D. O. (2016). Prevalência de ansiedade e depressão nos estudantes de Medicina da Universidade Federal do Pará. [Dissertação]. Belém: Faculdade de Medicina, Universidade Federal do Pará.

Babor, T. F., Higgins-Biddle, J., Saunders, J. \& Monteiro M. (2001). The alcohol use disorders identification test: guidelines for use in primary care. Genebra: World Health Organization, (2a ed.), https://www.who.int/publications/i/item/audit-the-alcohol-use-disorders-identification-test-guidelines-for-use-in-primaryhealth-care. 
Baldisserotto, C. M., Filho, E. S., Nedel F. \& Sakae T. M. (2005). Problemas psiquiátricos menores e indicadores do uso problemático de álcool entre os estudantes de medicina da Universidade do sul de Santa Catarina-UNISUL. Arq. Catarin. Med, 34:73-79. http://www.acm.org.br/revista/pdf/artigos/308.pdf.

Barbosa, F. L., Barbosa, R. L., Barbosa, M. C. L., Aguiar, D. L., Figueiredo, I. A., Ribeiro, A. C. et al. (2013). Uso de álcool entre estudantes de medicina da Universidade Federal do Maranhão. Rev. bras. educ. med., 37:89-95. http://dx.doi.org/10.1590/S0100-55022013000100013.

Becker, H. C. (2017). Influence of stress associated with chronic alcohol exposure on drinking. Neuropharmacology, 122:115-126. https://doi.org/10.1016/j.neuropharm.2017.04.028.

Brasil. Ministério da Saúde. (2019). Consumo abusivo de álcool aumenta 42,9\% entre as mulheres. Recuperado de: www.saude.gov.br/noticias/agenciasaude/45613-consumo-abusivo-de-alcool-aumenta-42-9-entre-as-mulheres\#: :text=Dados\%20in\%C3\%A9ditos \%20do\%20Minist\%C3\%A 9rio\%20da,2006\% $20(15 \% 2 \mathrm{C} 6 \% 25)$.

Bray, F., Ferlay, J., Soerjomataram, I., Siegel, R. L., Torre, L. A. \& Jemal, A. (2018). Global cancer statistics 2018: GLOBOCAN estimates of incidence and mortality worldwide for 36 cancers in 185 countries. CA Cancer J Clin, 68(6):394-424. https://doi.org/10.3322/caac.21609.

Carlini, E. A. (2011). Livreto informativo sobre drogas psicotrópicas. 6th ed. CEBRID: Centro Brasileiro de Informações sobre Drogas Psicotrópicas.

Carvalho, A. F., Heilig, M., Perez, A., Probst, C. \& Rehm J. (2019). Alcohol use disorders. The Lancet., 394:781-792. https://doi.org/10.1016/s01406736(19)317775-1.

Erol, A. \& Karpyak V. M. (2015). Sex and gender-related differences in alcohol use and its consequences: Contemporary knowledge and future research considerations. Drug Alcohol Depend., 156:1-13. https://doi.org/10.1016/j.drugalcdep.2015.08.023.

Galduróz, J. C. F., Noto, A. R., Nappo, S. A. \& Carlini E. A. (2005) Uso de drogas psicotrópicas no Brasil: pesquisa domiciliar envolvendo as 107 maiores cidades do país - 2001. Rev Latino-am Enfermagem, 13:888-95. https://www.scielo.br/pdf/rlae/v13nspe/v13nspea17.pdf

Gomes, V. L. O., Amarijo, C. L., Baumgarten, L. Z., Arejano, C. B., Fonseca, A. D. \& Tomaschewski-Barlem, J. G. (2013). Vulnerabilidade de estudantes de enfermagem e medicina pela ingestão de bebidas alcoólicas. Rev. enferm UFPE online, 7:128-34. https://doi.org/10.5205/reuol.3049-24704-1-LE.0701201318.

Gomes, L., Barroso, C., Silvestre, V., Baylão, A. C., Garcia, S. \& Pacheco S. J. (2018). Consumo de álcool entre estudantes de medicina do Sul Fluminense RJ. Rev. Med., 97:260-6. https://doi.org/10.11606/issn.1679-9836.v97i3p260-266.

Le Daré, B., Lagente, V. \& Gicquel, T. (2019). Ethanol and its metabolites: update on toxicity, benefits, and focus on immunomodulatory effects. Drug Metab Rev., 51:545-561. https://doi.org/10.1080/03602532.2019.1679169.

Martins-Oliveira, J. G., Jorge, K. O., Ferreira, R. C., Ferreira, E. F., Vale, M. P. \& Zarzar P. M. (2016) Risk of alcohol dependence: prevalence, related problems and socioeconomic factors. Ciênc. Saúde Coletiva, 21:17-26. http://doi.org/10.1590/1413-81232015211.00652015.

Mattara, F., Ângelo P., Faria J., Campos J. (2010). Confiabilidade do teste de identificação de transtornos devido ao uso de álcool (AUDIT) em adolescentes. SMAD. Revista Eletrônica Saúde Mental Álcool e Drogas, 6(2):296-14. http://pepsic.bvsalud.org/pdf/smad/v6n2/5.pdf.

Mendonça, A. K. R. H., Jesus, C. V. F. \& Lima, S. O. (2018). Fatores Associados ao Consumo Alcoólico de Risco entre Universitários da Área da Saúde. Rev. bras. educ. med., 42: 207-215. https://doi.org/10.1590/1981-52712018v42n1rb20170096.

Mourão, C. F., Andrade, B. H., Marques, C. F., Aparecida, V. M. \& Prates C. A. (2015). Fatores associados à prática do binge drinking entre estudantes da área da saúde. Rev. CEFAC, 17: 475-484. https://doi.org/10.1590/1982-021620158914.

Pedrosa, A. A. S., Camacho, L. A. B., Passos, S. R. L. \& Oliveira R. V. C. (2011). Consumo de álcool entre estudantes universitários. Cad. Saúde Pública, 27: 1611-1621. https://doi.org/10.1590/S0102-311X2011000800016.

Pelicioli, M., Barelli, C., Gonçalves, C. B. C., Hahn, S. R. \& Scherer J. I. (2017). Perfil do consumo de álcool e prática do beber pesado episódico entre universitários brasileiros da área da saúde. J. bras. Psiquiatr., 66: 150-156. https://doi.org/10.1590/0047-2085000000164.

Pereira, A. S., Shitsuka, D. M., Parreira, F. J., \& Shitsuka, R. (2018). Metodologia da pesquisa científica. UFSM. https://repositorio.ufsm.br/bitstream/handle/1/15824/Lic_Computacao_Metodologia-Pesquisa-Cientifica.pdf?sequence=1 .

Ramos, L. C. S. \& Cunha R. A. (2018). Perfil do consumo de álcool entre os estudantes de Medicina do Campus Lagarto da Universidade Federal de Sergipe [Dissertação]. Lagarto: Universidade Federal de Sergipe. https://ri.ufs.br/handle/riufs/9294.

Ribeiro, G. F. F., França, M. V., Faria, R. L. B. C., Cuellar, P. M. G. \& Martins M. L. B. (2015). Álcool: uso por estudantes de medicina da Universidade Federal do Tocantins. Revista Cereus, 7: 29-39. http://ojs.unirg.edu.br/index.php/1/article/view/841/334.

Santos, W. S., Gouveia, V. V., Fernandes, D. P., Souza, S. S. B. \& Grangeiro, A. S. M. (2012). Alcohol Use Disorder Identification Test (AUDIT): explorando seus parâmetros psicométricos. J. bras. Psiquiatr., 61: 117-123. http://doi.org/10.1590/S0047-20852012000300001.

Silva, E. C. \& Tucci A. M. (2014). Estudo transversal sobre o uso de risco de álcool em uma amostra de estudantes de uma universidade federal brasileira. $J$. bras. psiquiatr., 63: 317-325. https://doi.org/10.1590/0047-2085000000040.

Sompré I. (2019). Avaliação da qualidade de vida, da depressão e da ansiedade nos indígenas graduandos do curso de Medicina na Universidade Federal do Pará [Dissertação]. Belém: Faculdade de Medicina, Universidade Federal do Pará.

Spanagel R. (2018). Aberrant choice behavior in alcoholism. Science, 360:1298-1299. https://doi.org/10.1126/science.aau0668.

Spear L. P. (2018). Effects of adolescent alcohol consumption on the brain and behavior. Nature Reviews Neuroscience, 19:197-214. https://doi.org/10.1038/nrn.2018.10. 
Research, Society and Development, v. 10, n. 3, e58310313404, 2021

(CC BY 4.0) | ISSN 2525-3409 | DOI: http://dx.doi.org/10.33448/rsd-v10i3.13404

Spinelli, P. A., Valente, M. F. M. \& Lotério, H. A. (2009). Consumo de álcool e drogas pelos estudantes da Faculdade de Medicina de Jundiaí-SP. Perspectivas Médicas, 20: 19-25. https://www.redalyc.org/pdf/2432/243216397005.pdf.

Unidade de Pesquisa em Álcool e Drogas (UNIAD). Instituto Nacional de Ciência e Tecnologia para Políticas Públicas do Alcool e outras Drogas (INPAD). (2012) II Levantamento Nacional de Alcool e Drogas (LENAD). https://inpad.org.br/wp-content/uploads/2014/03/Lenad-II-Relat\%C3\%B3rio.pdf.

Zeferino, M. T., Hamilton, H., Brands, B., Wright, M. G. M., Cumsille, F. \& Khenti, A. (2015) Consumo de drogas entre estudantes universitários: família, espiritualidade e entretenimento moderando a influência dos pares. Texto Contexto Enferm, 24: 125-135. http://doi.org/10.1590/0104-07072015001150014. 